\title{
Einfluß von Gitterfehlern auf die dielektrischen Eigenschaften von Seignettesalz
}

\author{
Erich W. Labouvie \\ Institut für Experimentalphysik II der Universität des Saarlandes, Germany \\ (Z. Naturforsch. 23 a, 997-1000 [1968] ; eingegangen am 3. April 1968)

\begin{abstract}
It is shown by an etching method joined with microscopical observations that the structure of Rochelle salt crystals is not very much disturbed by dislocations and sudares. The correlation between the distributions of dislocations and sudares is obviously determined by the process of crystal growth. Measurements of the upper Curie-point and the dielectric constant are made in order to reveal some influence of these crystal defects on the dielectric properties.
\end{abstract}

Ziel dieser Arbeit soll es sein, die durch ein Ätzverfahren und lichtmikroskopische Beobachtungen gewonnenen Informationen über Sudares und Versetzungen mit Messungen des oberen Curie-Punktes und der maximalen Dielektrizitätskonstante (DK) zu vergleichen, um den Einfluß dieser eindimensionalen Gitterfehler auf die dielektrischen Eigenschaften von Seignettesalzkristallen direkt überprüfen zu können.

Messungen von Müser und $\mathrm{UnRUH}^{1}$ haben gezeigt, daß sich die verschiedenen Wachstumsgebiete in der Lage des Curie-Punktes, der Form der Hystereseschleife und der maximalen DK wesentlich unterscheiden. Untersuchungen über den Fremdstoffeinbau ${ }^{1-3}$ und das Auftreten von Sudares ${ }^{4-6}$ führten bisher noch zu keiner befriedigenden Erklärung dieser Tatsachen.

\section{Experimentelles}

Die untersuchten Kristalle wurden ausschließlich nach dem Temperatursenkverfahren ${ }^{7}$ aus einer übersättigten Lösung bei Temperaturen zwischen $28^{\circ} \mathrm{C}$ und $34{ }^{\circ} \mathrm{C}$ gezogen. Die Keime wuchsen bei gleichzeitiger Bewegung auf einer größeren Kreisbahn um die Mittelachse des Züchtungsgefäßes und gegenläufiger Eigenrotation um den Aufhängedraht in 2-4 Wochen zu $50 \mathrm{~g}$ schweren Blöcken. Die Kristalle wurden anschließend nach dem von $\mathrm{C}_{\mathrm{ADY}}{ }^{8}$ angegebenen Verfahren in etwa $3 \mathrm{~mm}$ dicke $a$-, $b$ - und $c$-Schnitte zerlegt, um Ätzbilder auf Schnittflächen aus verschiedenen Wachstumsgebieten vergleichen zu können. $\mathrm{Zu}$ diesem $\mathrm{Zwecke}$ wurden die Oberflächen auf einem weichen, leicht angefeuchteten Leder einige Sekunden lang poliert, geätzt und danach getrocknet. Nach den Untersuchungen

1 H. E. Müser u. H.-G. Unruh, Z. Phys. 162, 313 [1961].

2 A. G. Milligan, J. phys. Chem. 33, 1363 [1929].

3 V. P. Konstantinova u. V. A. Jurin, Soviet Phys.-Crystallography 2, 290 [1957].

4 H. Takahasi, T. Nakamura u. Y. Ishibashi, J. Phys. Soc. Japan 15, 853 [1960]. der Ätzpit- und Sudare-Verteilungen wurden den Kristallscheiben kleine $a$-Schnitte entnommen, deren Domänenkonfigurationen im Polarisationsmikroskop beobachtbar waren. Diese Proben wurden dann mit Blattgoldelektroden belegt und 6 Wochen bei $44 \%$ relativer Luftfeuchtigkeit gelagert. Die Messung der Kapazitäten in Abhängigkeit von der Temperatur erfolgte mit einer Brücke „General Radio Typ 1620-A“ bei einer maximalen Spannung von $50 \mathrm{mV}$ und einer Meßfrequenz von $1 \mathrm{kHz}$.

\section{Eigenschaften von Sudares}

Einige Zeit nach Entnahme aus dem Züchtungsgefä $\beta$ und Temperaturumstellung von etwa $30^{\circ} \mathrm{C}$ auf Zimmertemperatur werden in Seignettesalzkristallen weiße Schleier sichtbar (Abb. $1^{*}$ ), die aus einzelnen, in $c$-Richtung weisenden röhrenförmigen Einschlüssen, sogenannten Sudares ${ }^{5}$, bestehen. Sie sind im Mittel $10^{-3} \mathrm{~mm}$ dick und bis zu $2 \mathrm{~mm}$ lang. Eine Lagerung bei verschiedenen Luftfeuchtigkeiten zeigt keinen Einfluß auf das Entstehen dieser Fehler.

Dagegen kann aber festgestellt werden, daß das Sichtbarwerden der Einschlüsse verhindert wird, solange die Kristalle bei ihrer Züchtungstemperatur und mittlerer Luftfeuchtigkeit gelagert werden. Eine anschließende Temperatursenkung hat immer zur Folge, daß die weißen Schleier kurze Zeit später auftreten.

Entgegen früheren Beobachtungen und Vorstellungen ${ }^{4-6}$ können bei den hier verwendeten Kristallen keine charakteristischen Domänenbilder in unmittelbarer Umgebung der Sudares gefunden werden. Untersuchungen an Übergangszonen zwischen

5 T. Nakamura u. K. Ohi, J. Phys. Soc. Japan 16, 209 [1961].

6 K. Ohi u. T. Nakamura, J. Phys. Soc. Japan 17, 1195 [1962].

7 H. Flunkert, Diplomarbeit, Universität Münster 1957.

8 W. G. CAdy, Piezoelectricity, McGraw-Hill, New York 1946.

* Abb. $1-3$ und 5 auf Tafel S. 998 a, b. 
verschiedenen Wachstumsgebieten zeigen ebenso, daß auch der bevorzugte Aufbau von $b$-Domänen im $\{001\}$-Gebiet und von $c$-Domänen in den übrigen Gebieten nicht durch das Auftreten von Sudares bedingt sein kann (Abb. 2).

\section{Versetzungen in Seignettesalzkristallen}

Das oben genannte Ätzverfahren liefert auf $a$-, $b$ und $c$-Flächen gut reproduzierbare Ätzbilder. Die Ätzpits sind pyramidenförmige Vertiefungen mit einem rechteckigen, für jede der drei Flächen charakteristischen Grundriß. Aus der Tatsache, daß die Pitverteilungen auf sich entsprechenden Schnittflächen selbst bei größerem Abstand außerordentlich gut übereinstimmen, darf der Schluß gezogen werden, daß es sich hier um Versetzungen handelt, die vorwiegend geradlinig parallel zu den drei kristallographischen Richtungen verlaufen (Abb. 3).

Für die weiteren Untersuchungen interessant ist jedoch nur die Pit-Verteilung auf $c$-Flächen; die Ätzbilder auf den beiden anderen Flächen zeigen nämlich, daß sich die Versetzungen in $a$ - und $b$ Richtung mit einer Dichte von $10^{3} \mathrm{~cm}^{-2}$ auf eine schmale Zone in unmittelbarer Nähe von Keim und Aufhängedraht beschränken (Abb. 4). Es ist daher offensichtlich, daß, wenn überhaupt ein Einfluß auf die dielektrischen Eigenschaften erwartet wird, dieser nur von den Versetzungen in $c$-Richtung herrüh-

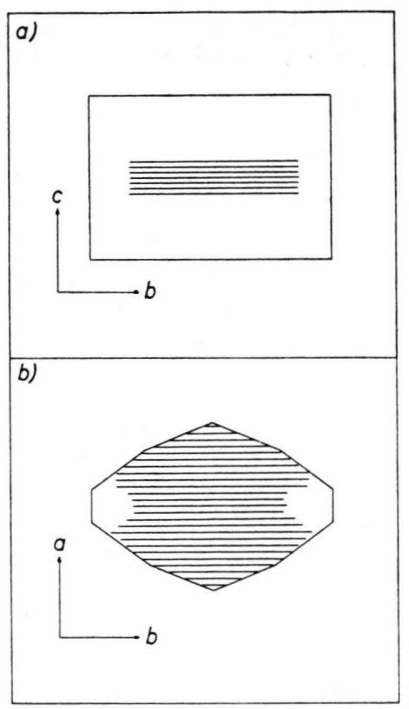

Abb. 4. Schematische Darstellung der Pit-Verteilungen a) auf (100) -Flächen [ähnliche Ätzbilder erhält man auf (010)-Flächen], b) auf (001)-Flächen. Gebiete mit hoher Pit-Dichte sind schraffiert. ren kann, weil sie sich mit Ausnahme des $\{010\}$ Gebietes über die gesamte Fläche eines $c$-Schnittes verteilen (Abb. 4).

Typische Ätzpit-Anordnungen auf $c$-Flächen zeigt die Abb. 5. Die mittlere Pit-Dichte schwankt zwischen $5 \cdot 10^{3}$ und $10^{4} \mathrm{~cm}^{-2}$. Der Versuch, durch plastische Verformung die Versetzungsdichte zu erhöhen, scheiterte an der Sprödigkeit des Materials.

Der Vergleich der Pit- und Sudare-Verteilungen ergibt, daß in unmittelbarer Umgebung eines Sudares stets mehrere Ätzpits und damit Versetzungen auftreten. Da die Pit-Verteilung bereits vor Sichtbarwerden der Sudares fixiert werden kann und sich nach ihrem Erscheinen nicht mehr ändert, liegt der Schluß nahe, daß der vermehrte Einbau von Versetzungen um Sudares im Wachstumsprozeß begründet liegt. Die Möglichkeit, daß es sich bei Sudares um allmählich aufgeweitete Versetzungskerne handelt, kann auf Grund der großen Abmessungen der Fehler ausgeschlossen werden.

\section{Dielektrische Messungen}

Eine direkte Ergänzung der vorgenannten Beobachtungen liefern Messungen des oberen CuriePunktes und der maximalen DK von kleinen Kristallproben aus verschiedenen Wachstumsgebieten. Verglichen werden jeweils zwei Proben aus einem Gebiet, die sich bezüglich der Dichte der Versetzungslinien in $c$-Richtung um drei Größenordnungen unterscheiden. Die Genauigkeit der Kapazitätsmessungen beträgt $\pm 2 \mathrm{pF}$. Die Bestimmung der Probenabmessungen führt zu einem relativen Fehler der DK-Werte von maximal 8\%. Beim Vergleich der beiden Proben eines zusammengehörenden Paares sind Temperaturunterschiede bis auf $\pm 0,01{ }^{\circ} \mathrm{C}$ genau bestimmt, beim Vergleich verschiedener Probenpaare bis auf $\pm 0,05^{\circ} \mathrm{C}$. Abb. 6 zeigt einige Meßergebnisse. Aufgetragen ist die reziproke DK über der Temperatur für die verschiedenen Wachstumsgebiete. Für das $\{010\}$-Gebiet ist nur eine Kurve eingetragen, da in diesem Gebiet keine Versetzungen auftreten. Die Werte der DK am CuriePunkt liegen zwischen 3600 und 6200, sind also relativ niedrig.

Die Reihenfolge der Wachstumsgebiete bezüglich ihrer Curie-Punktslage und der Größe der maximalen DK stimmt mit der von Müser und $\mathrm{UnRUH}^{1}$ gefundenen überein. Die absoluten Temperaturen der Curie-Punkte lassen sich auch gut in Messungen von 
Erich W. Labouvie, Einfluß von Gitterfehlern auf die dielektrischen Eigenschaften von Seignettesalz (S. 997).

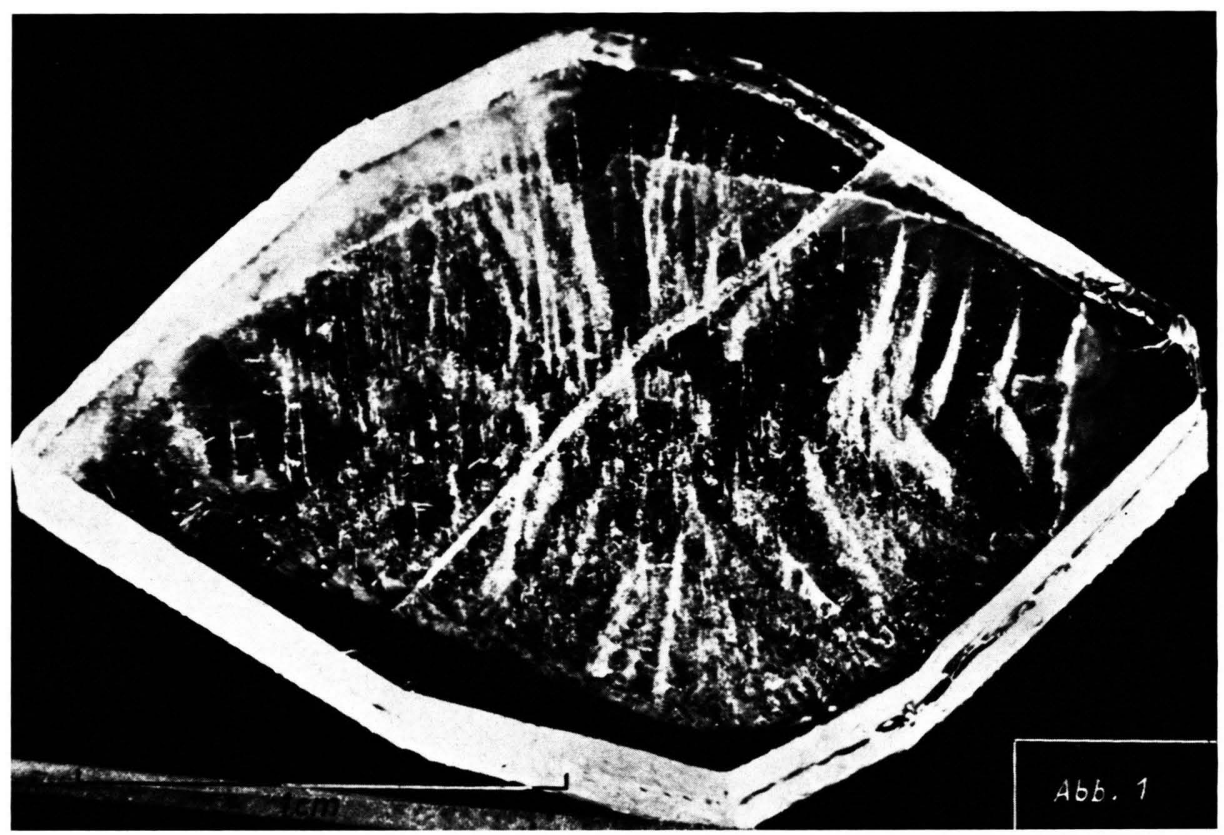

Abb. 1. Sudares in einem $c$-Schnitt, sichtbar als weiße Schleier.
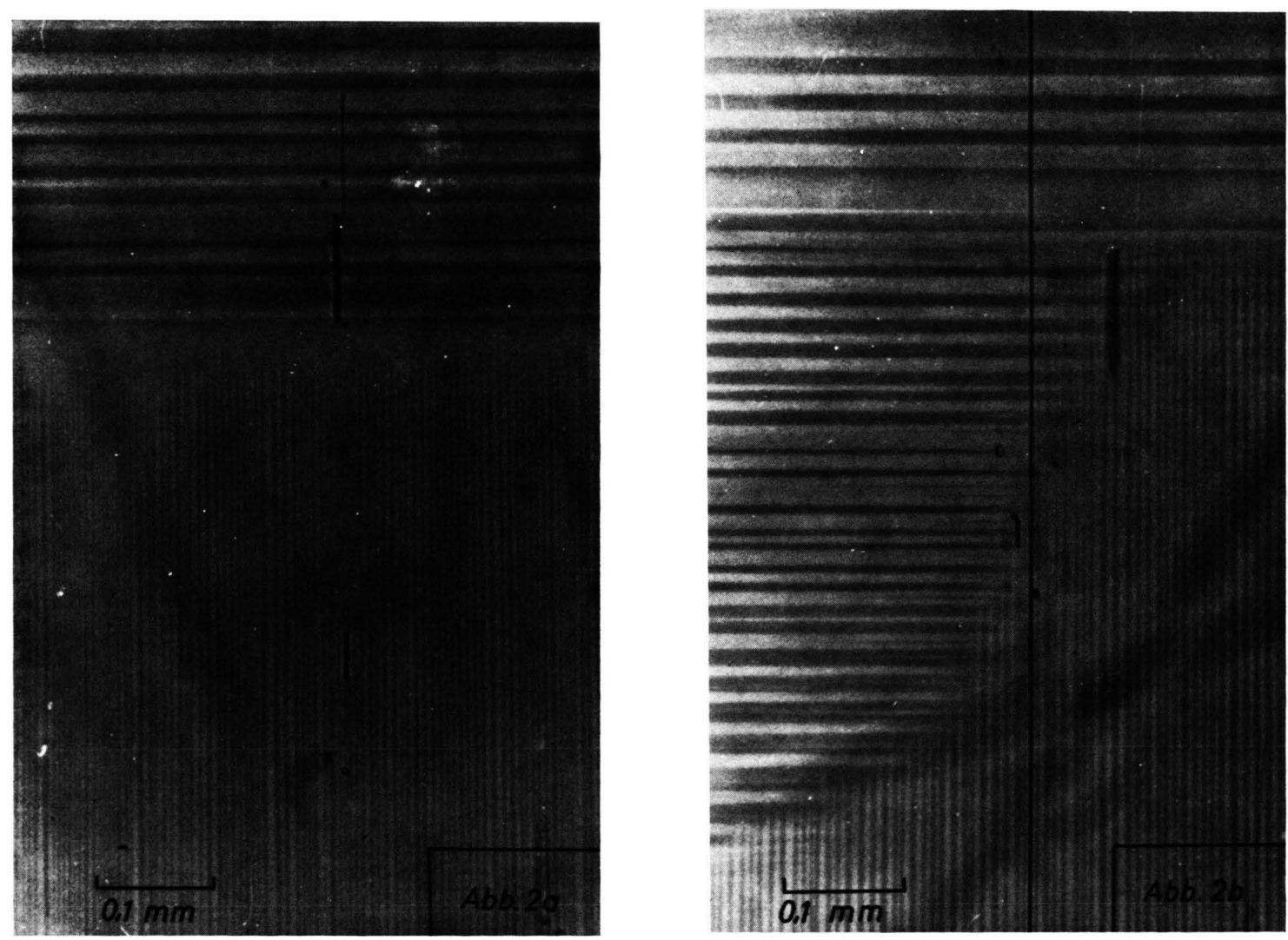

Abb. 2. Sudares in Übergangszonen zwischen $\{001\}$ - und $\{210\}$-Gebieten. 


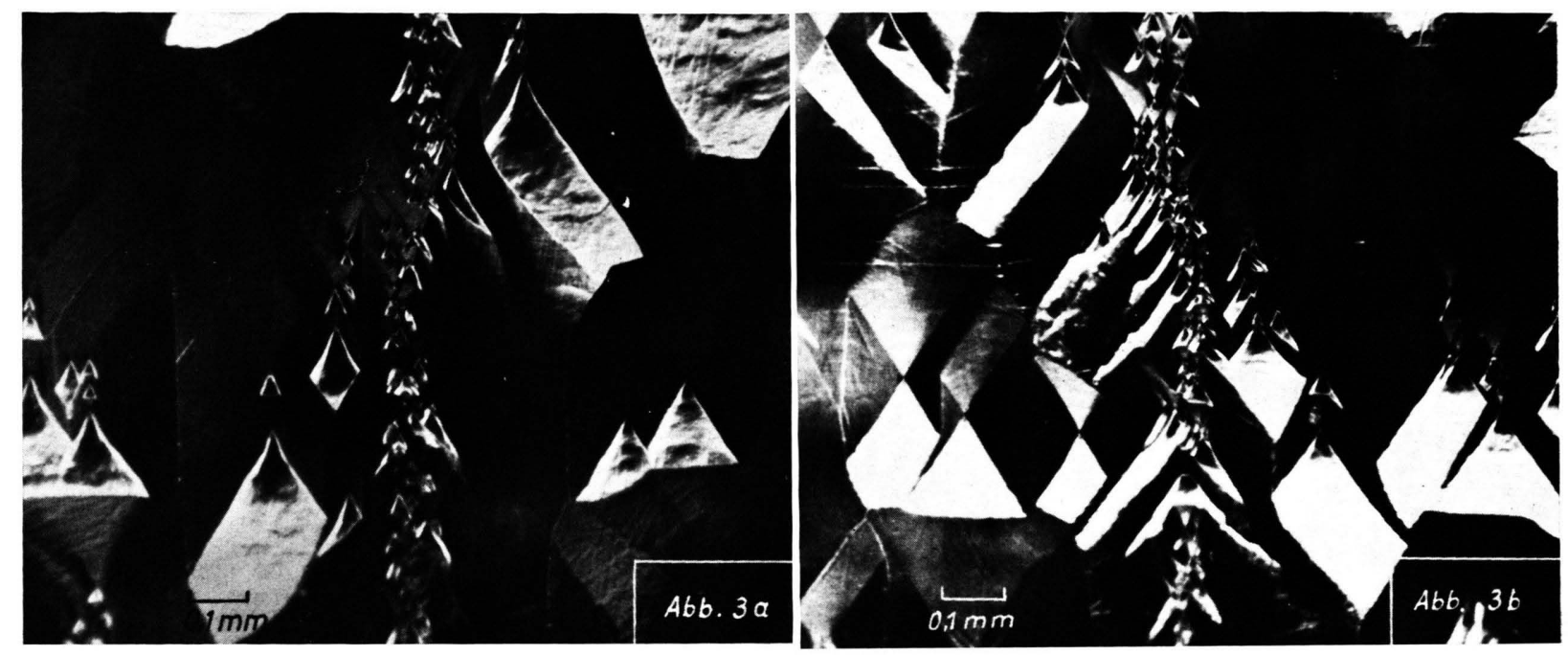

Abb. 3. Korrespondierende Pit-Verteilungen auf gegenüberliegenden $c$-Schnittflächen; Abstand etwa 1 mm.

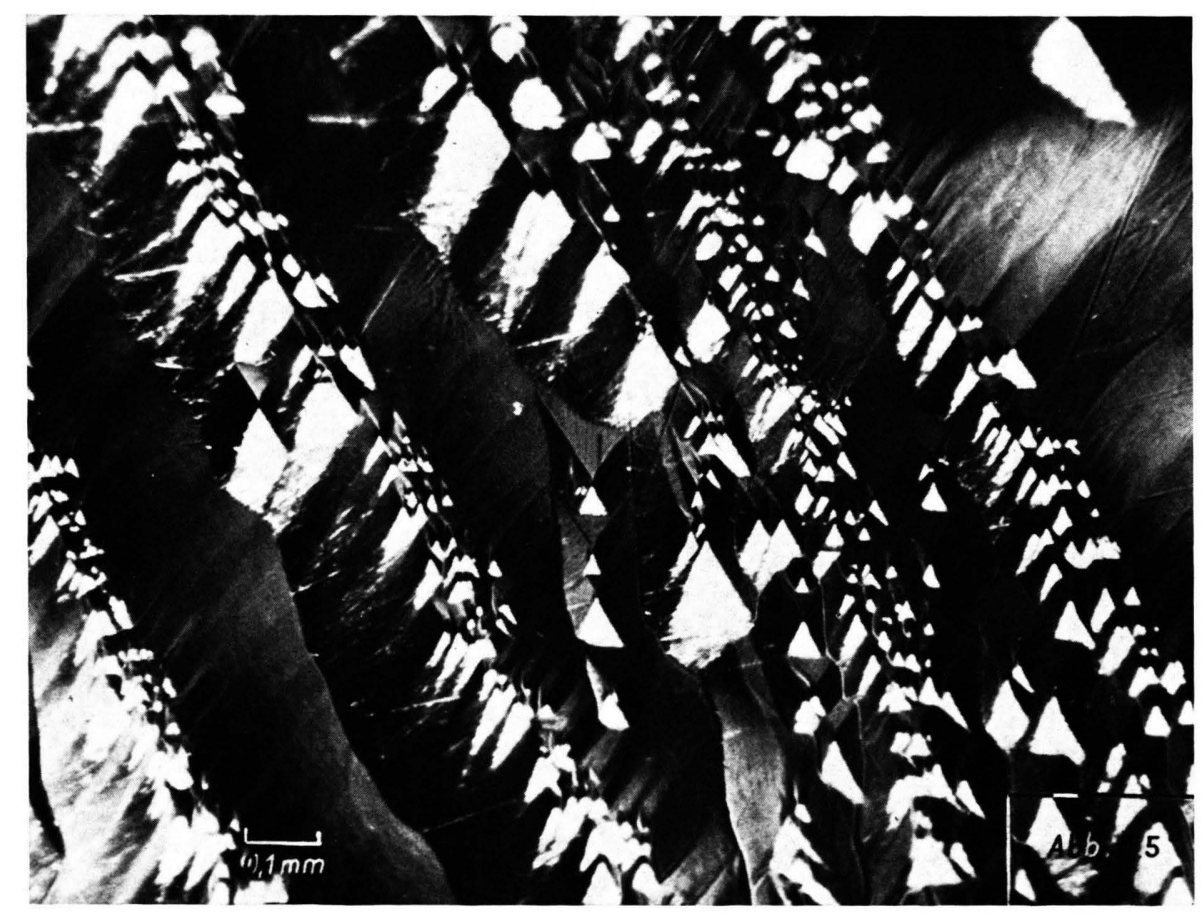

Abb. 5. Charakteristische Ätzpit-Anordnungen auf $c$-Flächen. 
Weissbach und Müser ${ }^{9}$ einordnen. Die Meßkurven zeigen eindeutig, daß in keinem Wachstumsgebiet durch Versetzungen oder Sudares signifikante Verschiebungen des Curie-Punktes hervorgerufen wer-

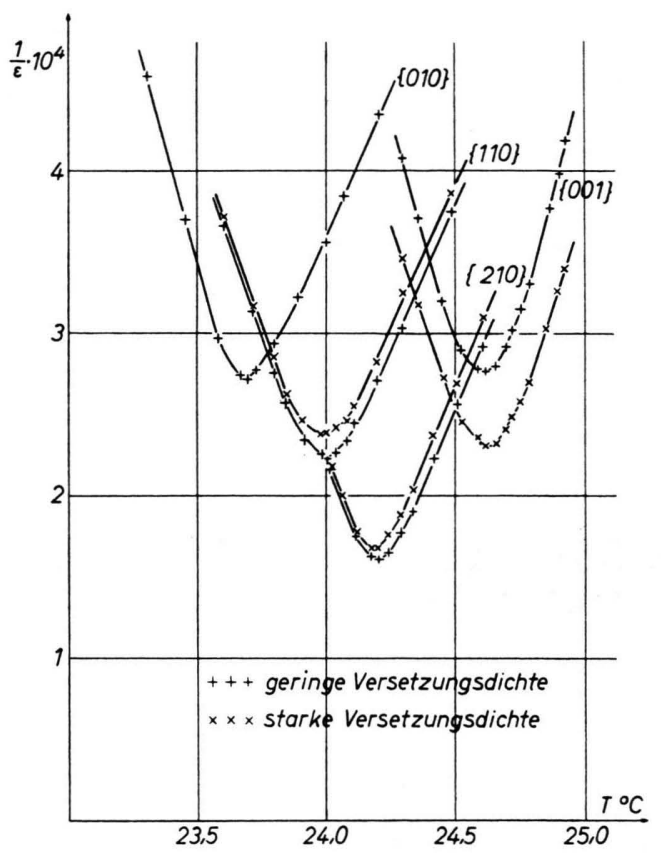

Abb. 6. Temperaturverlauf der reziproken DK in verschiedenen Wachstumsgebieten, gemessen an Kristallproben mit hoher und niedriger Versetzungsdichte.

den. Diese Tatsache wird noch verdeutlicht durch Messungen an Proben, die teilweise das $\{210\}$ - und $\{001\}$-Gebiet enthalten. In Abb. 7 ist schematisch der Verlauf der Versetzungslinien und der Grenze

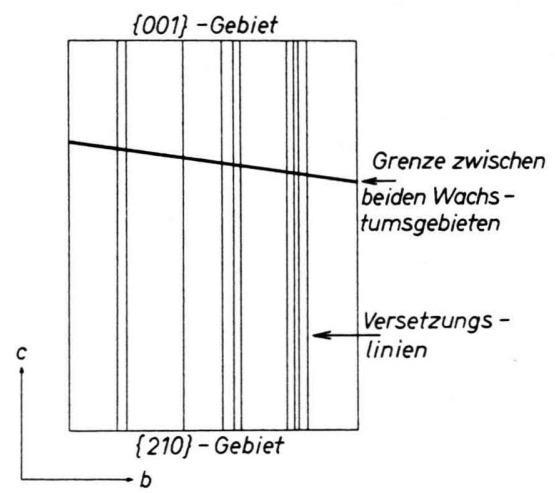

Abb. 7. Verlauf der Versetzungen in Kristallproben, die gleichzeitig das $\{210\}$ - und $\{001\}$-Gebiet enthalten.

9 G. Weissbach u. H. E. Müser, Phys. Status Solidi 13, 45 [1966]. zwischen den beiden Wachstumsgebieten in diesen Proben angedeutet. Die Meßkurven zu diesen Proben sind in Abb. 8 dargestellt und zeigen deutlich zwei verschiedene Curie-Punkte, obwohl sich die Wachstumsgebiete in ihrer Versetzungsdichte nicht unterscheiden.

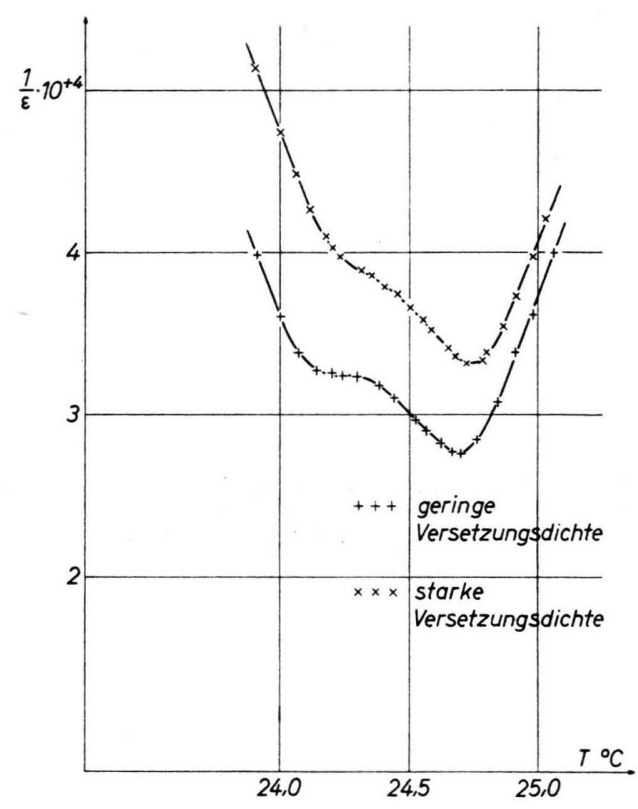

Abb. 8. Temperaturverlauf der reziproken DK von Kristallproben, die zwei Wachstumsgebiete enthalten (siehe Abb. 7).

\section{Diskussion der Ergebnisse}

Die Untersuchungen führen zu der Folgerung, da $\beta$ der Wachstumsproze $\beta$ auf (210) - und (110). Flächen sehr störempfindlich ist. Offenbar werden auf diesen Flächen röhrenförmige Lösungseinschlüsse beim Wachstum gebildet, in denen bei einem Temperaturabfall eine irreversible Auskristallisation einsetzt, die dazu führt, daß die Fehler als Sudares sichtbar werden. Da die tangentielle Wachstumsgeschwindigkeit in $c$-Richtung relativ groß ist, ist $\mathrm{zu}$ erwarten, daß entstehende Störungen sich gerade in dieser Richtung ausdehnen. In der nächsten Umgebung eines Einschlusses ist das Gitter auf Grund des unterschiedlichen elastischen Verhaltens stark verspannt. Um diese Spannungen zu vermindern, werden gerade hier leichter und in verstärktem Maße Versetzungen eingebaut werden.

Die polarisationsoptischen Beobachtungen und dielektrischen Messungen lassen erkennen, daß Sudares und Versetzungen keineswegs solche Span- 
nungs- und Deformationsfelder besitzen, die die ferroelektrische Polarisation in $a$-Richtung beeinflussen. Somit liegt der Schluß nahe, daß es sich vorwiegend um Stufenversetzungen handelt. Die Piezokoeffizienten nämlich, die die dabei auftretenden Deformationen $S_{1}, S_{2}$ und $S_{6}$ mit der Polarisation $P_{1}$ in der ferroelektrischen Richtung koppeln, verschwinden im parelektrischen Bereich ganz; im ferroelektrischen Bereich verschwinden sie zum Teil ebenfalls, oder sie sind außerordentlich klein.

Die Bildung von Schraubenversetzungen in $c$ Richtung ist aus energetischen Gründen weniger wahrscheinlich. Die Polarisation $P_{1}$, die durch die Scherung $S_{4}$ hervorgerufen wird, müßte entspre- chend dem Deformationsfeld am Ort der Versetzung ihr Vorzeichen umkehren, wodurch starke Raumladungen mit Streufeldern entständen.

Die dielektrischen Messungen machen überdies deutlich, daß die Wachstumsgebiete durch relativ scharfe Grenzen ohne Übergangszonen voneinander getrennt sind. Ihre unterschiedlichen Eigenschaften können nicht auf einen Einfluß der oben genannten Fehler zurückgeführt werden.

Herrn Prof. Dr. H. E. Müser danke ich für die Anregung zu dieser Arbeit und die Bereitstellung von Institutsmitteln, Herrn Dr. G. Berndes für seine Hilfe bei der Durchführung der Untersuchungen.

\title{
Berechnung der komplexen atomaren Streufaktoren für schnelle Elektronen unter Verwendung von Hartree-Fock-Atompotentialen
}

\author{
(2. Mitteilung) \\ JoAChim HaAse \\ Lehrstuhl für Physikalische Chemie der Universität Ulm *, Ulm \\ (Z. Naturforsch. 23 a, 1000-1019 [1968] ; eingegangen am 9. Januar 1968)
}

\begin{abstract}
In der vorliegenden Arbeit wird über die Berechnung der komplexen Streufaktoren für schnelle Elektronen an sechs Atomen unter Verwendung von Hartree-Fock-Potentialen bei fünf verschiedenen Beschleunigungsspannungen nach der Partialwellenmethode berichtet. Zur Berechnung der Werte wurde ein Näherungsverfahren verwendet, das sich aus der WBK-Methode ableiten läßt. Konvergenzkorrekturen für die Reihensummierung des Real- und Imaginärteils der komplexen Streufaktoren sind angegeben. Die Einflüsse des Näherungsverfahrens, der Anzahl der zur Summierung verwendeten Partialwellenphasen, sowie der Konvergenzkorrekturen werden diskutiert. Um die in Tabellenform vorliegenden Werte der komplexen Streufaktoren zur Auswertung von Elektronenbeugungsaufnahmen von Molekülen im Gaszustand besser verwenden zu können, wurden Beträge und Phasen der Streufaktoren durch analytische Ausdrücke angepaßt und die zur Approximation notwendigen Koeffizienten angegeben.
\end{abstract}

Zur Strukturbestimmung freier Moleküle mit Hilfe der Methode der Elektronenbeugung an Gasen benötigt man, wie erstmals von Schomaker und Glau${ }_{\text {BER }}{ }^{1}$ gezeigt werden konnte, genaue Werte der komplexen atomaren Streufaktoren. Wie diese Autoren zeigen konnten, gelang es unter Verwendung der Streufaktoren in erster Bornscher Näherung ${ }^{2}$ nicht, bei der Bestimmung der Struktur des gasförmigen $\mathrm{UF}_{6}$ zu einem eindeutigen Ergebnis zu kommen.

Führt man an Stelle der Formfaktoren in erster Bornscher Näherung $f^{\mathrm{B}}(s)$ die komplexen Streufak.

* Derzeitige Anschrift: 75 Karlsruhe, Hertzstr. 16, Bau 35.

1 V. Schomaker u. R. Glauber, Nature London 170, 290 [1952]; - Phys. Rev. 89, 667 [1953]. toren $f(s)=|f(s)| \exp (i \eta(s))$ in den Ausdruck für die molekulare Intensitätsfunktion, die, aus der kinematischen Streutheorie folgend, die Winkelabhängigkeit der an gasförmigen Molekülen gestreuten Elektronen angibt, ein, so erhält man folgende Formel

$$
\begin{aligned}
I_{\mathrm{m}}(s)= & \sum_{\substack{i \\
i \neq j}} \sum_{\substack{j \\
i \neq j}}\left|f_{i}(s)\right|\left|f_{j}(s)\right| \cos \left(\eta_{i}(s)-\eta_{j}(s)\right) \\
& \cdot \exp \left(-l_{i j}^{2} \frac{s^{2}}{2}\right) \cdot \frac{\sin s \cdot r_{i j}}{s r_{i j}} .
\end{aligned}
$$

${ }^{2}$ M. Born, Z. Phys. 37, 863 [1926] ; 38, 803 [1926]. 